\title{
ECS-induced retrograde amnesia for one trial active avoidance'
}

\author{
DONALD J. LEWIS, RALPH R. MILLER, JAMES R. MISANIN, AND NORMAN G. RICHTER
}

A procedure is described for obtaining one trial active avoidance in a standard step down apparatus. Four groups of rats were given a single step down trial consisting of either platform shock, platform shock followed by ECS, ECS, or no treatment. On the test trial, the platform shock Ss stepped off the platform significantly faster than the other groups which did not differ among themselves. The data refutes the contention that ECS acts selectively on passive as opposed to active responses.

When electroconvulsive shock (ECS) is delivered soon after the acquisition of a response, a performance deficit for that response, called retrograde amnesia, is frequently observed. The prevalent explanation of this amnesia is that ECS acts as a strong interfering stimulus which disrupts the "consolidation" of a still unstable neural engram (e.g., McGaugh, 1966). In recent years, this theory has had to contend with several alternative hypotheses (e.g., Coons \& Miller, 1960; Lewis \& Maher, 1965). Before the controversy can be resolved, however, the conditions under which ECS will induce an apparent amnesia must be made explicit. For this reason, current interest has focused upon single training trial-single ECS paradigms which minimize the uncertainty of the age of the engram encountered in multiple trial studies and eliminate the conditioned fear and numerous unconditioned effects of multiple ECS.2

The large majority of retrograde amnesia studies using a one trial-one ECS paradigm have been limited to passive avoidance situations. 3 The restriction has been due not to a failure to produce amnesia for active avoidance, but to the lack of a consistently learned one trial active avoidance task. This omission in the data occasionally has been misinterpreted to infer that ECS acts selectively upon passive avoidance responses or, more particularly, upon CERs which are indistinguishable from discriminated avoidance in the passive situation. Such a selectivity may hold in multiple ECS paradigms (Gerbrandt \& Thomson, 1964) but the relevant data from single ECS studies have been lacking.

The present study was conducted to determine if a single ECS would produce amnesia for a one trial active avoidance response. The guiding principle was to create a paradigm analogous to the one trial passive avoidance studies performed in a standard step down apparatus (Jarvik \& Essman, 1960), a situation in which ECS is known to create amnesia (e.g., McGaugh,
1966). Pilot work to obtain a one trial active response started by simply applying platform shock in analogy to the floor shock of the passive paradigm. Contrasted with no shock Ss, the platform shock group yielded longer step down latencies. The problem appeared to be that the platform shock lasted only a few tenths of a second before the rat was "knocked off" the platform as opposed to the 5 sec floor shock used in our laboratory in the passive situation. This short aversive stimulus did not give the $\mathrm{S}$ time to orient and, hence, only produced a CER. The current study remedied this problem by introducing a removable shield around the platform that prevented early escape from the electrified platform.

Method

The Ss consisted of four groups of 15 male, albino Sprague-Dawley (Madison, Wis.) rats, 160-180 $\mathrm{g}$ in weight and 80-100 days old. Each rat was individually caged and maintained on ad lib water and $10 \mathrm{~g}$ Purina rat chow powder per day.

The step down apparatus consisted of a 23 in. deep open top box with a $20 \times 20$ in. grid floor. Centered in the box was a $4 \times 6-1 / 2$ in. grid platform $3-1 / 2$ in. above the floor. Suspended $18 \mathrm{in}$. over the platform was a 21 in. tall, hollow, rectangular wooden shield, 4-1/2 $x$ 7 in., that could be lowered over the platform preventing a rat on the platform from stepping down. Earclips for ECS administration were worn by all Ss on each occasion in the apparatus. The earclip wires passed up through the shield to a counterweighted pulley system designed to minimize the earclips as a distraction. Platform shock was provided by a Grason-Stadler E1065GS shock generator set at $1.6 \mathrm{~mA}$. ECS was a $40 \mathrm{~mA}$ average square wave that produced a full tonic-clonic seizure in all instances.

For two days before training, all Ss received two daily earclip adaptation trials of 2 min each in their home cages. On the treatment day, Group PS Ss were placed in the shield on the platform. After $2 \mathrm{sec}$ the platform was electrified. Five sec later, the shield was raised allowing the $S$ to escape to the floor from which he was returned to his home cage after $5 \mathrm{sec}$. The other three groups received identical treatments except that Group N received no platform shock, Group PS + ECS received ECS after escape and $5 \mathrm{sec}$ on the floor. Group ECS received no platform shock but ECS 5 sec after the step down response. Twenty-four $h$ after treatment, each $S$ was placed in the shield on the platform for $7 \mathrm{sec}$, after which time the shield was 


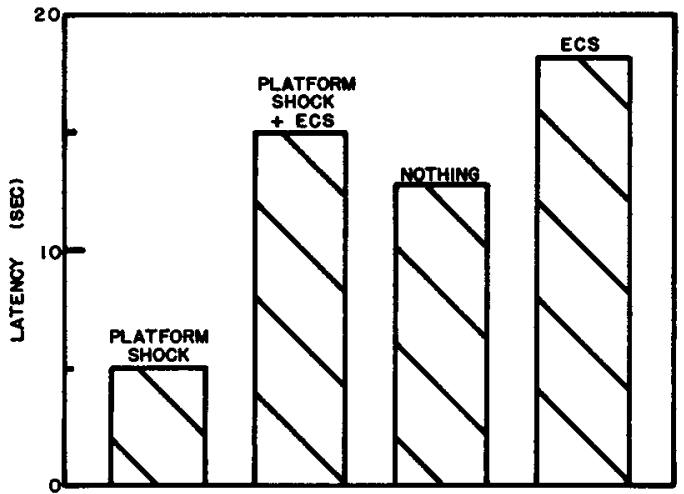

Fig. 1. Mean step down latencies on the $24 \mathrm{~h}$ test. The platform shock group differed $(p<.01)$ from each of the other groups which did not differ among themselves.

raised and the S's subsequent step down latency was recorded. An identical test was performed $48 \mathrm{~h}$ after training.

\section{Results and Discussion}

Mean step down latencies are illustrated in Fig. 1. The latencies were approximately normalized by a reciprocal transformation. An analysis of variance on reciprocal latencies proved significant (overall $\mathrm{F}=$ 15.34, df $=3 / 56, p<.01$ ), so a Newman-Keuls range test was performed (Winer, 1962). Group PS was found to differ significantly from each of the other three groups $(q(E C S)=5.43, q(N)=4.97, q(P S+E C S)=4.34, d f=1 / 56$, ps $<.01$ ) which did not differ significantly among themselves ( $p s>.05$ ). The $48 \mathrm{~h}$ test was entirely consistent with the $24 \mathrm{~h}$ test.

The difference between Groups PS and $\mathrm{N}$ is evidence for successful one trial active avoidance, and a comparison of Groups PS and PS + ECS indicates that ECS produced a complete behavioral amnesia for the learning trial. The possibility that this behavioral deficit is an ECS-alone effect, conditioned or unconditioned, is unlikely due to the similarity of the ECS and N groups. However, the nonsignificant trend toward longer latencies going from Group N to PS+ ECS to ECS suggests that ECS alone does have some slowing effect in this situation, similar to the slowing effect of ECS in the standard passive step down situation reported by Miller \& Lewis (1967).

The results of this study show that a single ECS has a comparable effect on both active and passive avoidance. It appears doubtful that the Gerbrandt \& Thomson (1964) hypothesis that ECS acts selectively on passive avoidance responses or, more particularly, upon CERs, can encompass the single ECS paradigms. ECS may in fact act differentially on different types of responses, but this difference is not an all-or-none phenomenon in respect to the active-passive distinction. It is too early to attempt to formulate a comprehensive explanation of the behavioral effects of ECS, but it seems doubtful that a single elementary explanation will suffice.

\section{References}

Coons, E. E., \& Miller, N. E. Conflict versus consolidation of memory traces to explain "retrograde amnesia" produced by ECS. J. comp. physiol. Psychol., 1960, 53, 524-531.

Gerbrandt, L. K., \& Thomson, C. W. Competing response and amnesic effects of electroconvulsive shock under extinction and incentive shifts. J. comp. physiol. Psychol., 1964, 58, 208-211.

Jarvik, M. E., \& Essman, W. B. A simple one-trial learning situation for mice. Psychol. Rep., 1960, 6, 290.

Lewis, D. J., \& Maher, B. A. Neural consolidation and electroconvulsive shock. Psychol. Rev., 1965, 72, 225-239.

McGaugh, J. L. Time-dependent processes in memory storage. Science, 1966, 153, 1351-1358.

Miller, R. R., \& Lewis, D. J. Electroconvulsive shock as a US in one-trial conditioning. Paper presented at Eastern Psychological Association, Boston, April, 1967.

Misanin, J. R. Sensitivity and reactivity to shock following electroconvulsive stimulation. Unpublished doctoral dissertation, Rutgers University, 1967.

Tenen, S. S. Retrograde amnesia from electroconvulsive shock in a one-trial appetitive learning task. Science, 1965, 148, 12481250.

Winer, B. J. Statistical principles in experimental design. New York: McGraw-Hill, 1962.

\section{Notes}

1. This research was supported by Research Grant MH-07 129 from the National Institute of Mental Health, United States Public Health Service.

2. Even a single ECS has been observed to have unconditioned nonamnesic consequences (Miller \& Lewis, 1967; Misanin, 1967), but these effects are usually sufficiently small that, with proper controls, they do not wash out the amnesic phenomenon.

3. Tenen (1966) has recently provided an example of ECS-induced amnesia for one trial appetitive learning. 